\title{
Comparative Evaluation of Various Techniques to Record Centric Relation- A Literature Review
}

\author{
Manoj Shetty ${ }^{1}$, Ganaraj Shetty² \\ ${ }^{1}$ Department of Oral Implantology, A. B. Shetty Memorial Institute of Dental Sciences, NITTE \\ (Deemed to be University) Deralakatte, Mangalore, Karnataka, India. ${ }^{2}$ Department of \\ Prosthodontics and Crown \& Bridge, A. B. Shetty Memorial Institute of Dental Sciences, NITTE \\ (Deemed to be University) Deralakatte, Mangalore, Karnataka, India.
}

\section{ABSTRACT}

\section{BACKGROUND}

The documentation of centric relation from the completely edentulous patient and transferring the same to the fully-adjustable articulator or semi-adjustable articulator is the most essential step, that helps in diagnosing, treatment planning, and fabrication of the complete denture prosthesis, that aids in enhancing the facial aesthetics, function, speech, and maintenance of the stomatognathic system. Recording of maxillomandibular relationship is the most critical step, the relation of mandible to maxilla creates more controversy than any other dental-related studies. Hence to re-establish the maxillomandibular relationship in a completely edentulous patient is the most difficult task. However, a historical literature review in dentistry reveals that there are numerous techniques, philosophies, and materials that can be manipulated and used to record the maxillomandibular relationship. The recording of centric and eccentric relationship of the mandible to maxilla can be done using various techniques like check bite record, intra-oral graphic recording, extra-oral graphic recording, functional recording, radiographic imaging and measurements based on anatomical landmarks, and computerized assessment of maxillomandibular relationship based on jaw movement tracking systems. The principle reason of recording maxillomandibular relationship in all the centric and eccentric relationship is to re-establish a maxillomandibular relationship without an error, as stated in literature review, that to initiate any treatment in dentistry it is must to record centric relation with proper guidance of mandibular jaw to maxillary jaw that should be in harmony with all the muscles of mastication and facial expression including the temporomandibular joint and bony structure, that helps to maintain the health and growth of the muscle tissues and bony structures. This article discusses the in-depth details of these techniques and methods used in recording centric jaw relations.

\section{KEY WORDS}

Centric Relation, Extraoral Tracing, Intraoral Tracing, Radiographic Assessment, Axiographic Assessment
Corresponding Author: Dr. Ganaraj Shetty, Senior Lecturer, Department of Prosthodontics and Crown \& Bridge, A. B. Shetty Memorial Institute of Dental Sciences, Nitte (Deemed to be University), Deralakatte,

Mangalore-575018, Karnataka, India.

E-mail:drganaraj@nitte.edu.in

DOI: $10.14260 /$ jemds/2020/12

Financial or Other Competing Interests: None.

How to Cite This Article:

Shetty M, Shetty G. Comparative Evaluation of Various Techniques to Record Centric Relation- A Literature Review. J. Evolution Med. Dent. Sci. 2020;9(01):53-59, DOI: 10.14260/jemds/2020/12

Submission 28-09-2019,

Peer Review 25-12-2019,

Acceptance 31-12-2019,

Published 06-01-2020. 


\section{BACKGROUND}

The relation of maxilla to the mandible creates more controversy than any other dental-related studies. Since this contestation is considered the base of all the disciplines in dentistry, the persuasion regarding all the techniques varies. Completely edentulous patients have poor neuromuscular proprioceptive guidance to navigate the mandible to the desirable centric relation; hence for the rehabilitation of the completely edentulous patient, a repeatable, recordable and learnable relationship of maxillary to the mandibular jaw is required.[1] The relation of maxilla to the mandible and to the condyles that articulate with the thinner most avascular part of the respective condylar discs with the complex in the anterior and superior most position against the glenoidal slopes of the articular eminences. This position is independent of incisal or occlusal contact. This position is clinically perceptible when the mandible is guided to the superior and anterior portion of the glenoid fossa. ${ }^{[2],[3],[4]}$ The centric and eccentric relationship of the mandible to maxilla can be recorded through various techniques like check bite record, graphic recording, functional recording, radiographic and computerized assessment of imaging centric relation. The principle reason to record centric relation is to establish a maxillomandibular relation without an error, as stated in literature review that to initiate any treatment in dentistry it is must/mandatory to record centric relation with proper guidance of mandible to maxillary arch that should be in harmony with all the muscles of mastication and facial expression including the joint and bone, that helps to maintain the anatomic and physiologic health of the muscles, tissues and bony structures.[2],[5],[6] This article discusses the various techniques and methods used in recording centric jaw relations that can aid in achieving the centric relation.

\section{DISCUSSION}

The significance of recording centric relation in the fabrication of complete denture prosthesis has been well known for many years. Some dental surgeons arrogate that a precisely recorded centric relation is the most appropriate measurement made in the fabrication of complete denture prosthesis. Various techniques and methods have been documented in the literature studies, some of the important techniques that gathered popularity in recording centric relation are, Direct check bite recording, Graphic intra-oral tracing, Graphic extra-oral tracing, Functional recording, Radiographic tracing method and Computerized tracing method. [7], [8]The historical review prevails that direct check bite techniques were the first technique used to record centric relation.[9] the technique is also known as a Physiologic Method. The patient's proprioception, the tactile sense and visual acuity of the dental surgeon is essential to document an accurate and precise centric relation record. This technique was introduced and described by pfaff, dentist of Fredrick in 1756, the technique of recording centric relation was the only technique, until the end of $19^{\text {th }}$ century. [10], [11] this technique was also known as non-precision jaw relation recording by placing a thermoplastic or rigid dental material, usually, wax or compound or dental plaster, between the rims, guiding the patient to close onto the material. This technique was also known as the Squash Bite technique. Hanau in 1923, Considered and modified the intraoral wax method. He pointed out the Resiliency of the tissue bearing, a denture-supporting area that might contribute to the distortion of the record. He, later on, advocated that recording of the positional relationships of maxilla to the mandible, under no pressure technique will minimize the error caused by Resiliency of the tissue. Hanau, with other researchers, agreed with the 'zero-pressure' philosophy, certain authors, disagreed with Hanau's philosophy they advocated the use of minimal pressure.[12],[13] There has been much criticism with inter occlusal "check-bites" records for Centric Relation. In 1932, Schuyler stated that the recording materials should be of uniform thickness and motion else it might be the reason for the errors in interocclusal records, which would cause an error in occlusion. He suggested that the modelling compound would be preferred as recording material because it can be softened more evenly, and does not release stress leading to distortion as much as wax. The interocclusal bite record secured on compound or wax rims are not sufficiently stable and free from common errors. Simpson concluded that records made with waxes were unsatisfactory, he stated that the technique of guiding the mandibular jaw back on closing, elevating the tongue, and having the patient swallow as he closes the jaw are the reason that they are unscientific and always carry with them the fallacy of guess or errors in recording centric relation. Payne and hickey stated that dental plaster as the most preferred material because of its rigidity and based on its setting time. In 1939. Wright further described factors that caused the distortion of interocclusal records are, salivary film, fit of denture bases, Resiliency of the underlying tissue, and the Pressure applied while recording the bite. $\mathrm{He}$ advocated that no pressure technique should be used to record centric relation. Later Brown in 1954, suggested, the centric relation can be obtained by having the patient bite repeatedly into softened wax rims, further, it was concluded that the centric relation obtained with these techniques was due to habitual motion of the joint and not the real centric. Greene used a new technique of breaking the memory of muscles and then recording the centric relation; he had the patience to hold the jaws apart for few seconds to fatigue the muscle tissues and then guided the patient to bite on the rims to record the centric relation. He then made orientation lines and troughs in the rims to re-orient them, following removal from the mouth. Gradually, the procedure evolved into interocclusal bite records as they are usually done today. Small amounts of wax, compound, dental plaster, Zinc-Oxide Eugenol Impression paste, the elastomeric impression material were placed between the occlusal rims, and the patient was asked to close the jaws into centric relation.[14],[15],[16] the advances were an attempt to reduce or equalize the occlusal pressure of vertical and horizontal contact. There are many opinions regarding the bite registration materials to use as inter-occlusal records "In 1955, Trapozzano stated that the wax can be used as one of the preferred material to record centric relation, later on, he 
termed it as a "Check-bite method" to record centric relation. Gysi in his study stated that he never obtained the same reading while recording centric from the same patient recording at a different time; He summarized that the elastic recovery of wax upon cooling will lead to the distortion. "Schuyler; stated that records made using impression compound were of great interest, the major disadvantage was the rigidity of compound material that leads to distortion of the records due to uneven or premature contact of the occlusal surface, due to uneven thickness of occluding rims, that may disturb the relation of the record bases. To overcome some of the disadvantages of check bite records they introduced a never technique to record the centric relation that was a type of intraoral and extraoral tracing mechanism which was introduced as a graphic tracing method of recording centric relation.[13],[17]

\section{Graphic Method of Recoding Centric Relation}

The gothic arch tracing device is commonly used in this technique, the device is attached to the maxillary and mandibular arches; the shape of the tracing depends on the relative movement of the mandibular jaw in centric and eccentric movements. It indicates the horizontal relation of the mandible to the maxillae. The apex of an accurately recorded tracing indicates the most retruded relation of the mandible to the maxillae from which lateral movements can take place. Graphic methods can be used as either intra-oral or extra-oral tracing Fig. 1. In 1866 Balkwill stated that the Gothic arch tracing depends on the various movements made by mandibular arch. In 1897, Heisse introduced "needlepoint tracing". The tracer made by GYSI was an extra-oral tracer. The tracing plate was coated with wax and attached to the mandibular occlusal rim. A spring-loaded pin will be mounted on the maxillary occlusal rim, to make the rims more stable it is recommended that the rims shall be made of modelling compound to maintain the vertical dimension of occlusion. The good tracing can be recorded, by guiding the patient to hold the rims in the apex of the tracing while notches were scored in the rims for orientation. Sears used lubricated rims for easier mandibular movement. He placed the needlepoint tracer on the mandibular rim and the plate on the maxillary rim. He stated that the angle formed by this technique of the tracing was more acute and easily discernible. Phillips: 1927, observed, The distortion of the record, caused due to right and left lateral mandibular movements, he introduced a plate for the maxillary occlusal rim and a tripoded ball bearing, mounted on a jackscrew for the mandibular occlusal rim.[18] This innovation was termed as "central bearing point", Stansbery then introduced a newer technique using a curved plate with a four-inch radius Corresponding to Monson's curve, mounted on the maxillary occlusal rim. A central bearing screw was attached to the plate of a three-inch radius curve Reverse-Monson curve on the mandibular occlusal rim. Following extra-oral tracing was made; a thin mix of dental plaster was injected between the maxillary and mandibular occlusal rims to form a biconcave centric registration.

Hall, in 1929, used Stansbery's method, substituted impression compound to obtain Centric Relation record.
Later, a graphic recording method used the central bearing point to produce the Gothic arch tracing. Later on, Hardy introduced "Coble Balancer". He then modified it in 1942, made a depression with a round bur at the formed apex of the tracing. The patient was advised to hold the bearing point in the depression while the dental plaster was injected in between the rims to make a centric relation record. Pleasure, in 1955, made use of a disk made up of plastics, attached them to the tracing plate with a hole over the apex of the Gothic arch. The Centric Relation record was made without any variation in the vertical dimension. The Sears, later on, introduced a tracer to record both intraoral and extraoral tracing together, the trivet that had an intra-oral central bearing point and two extra-oral tracing plates.

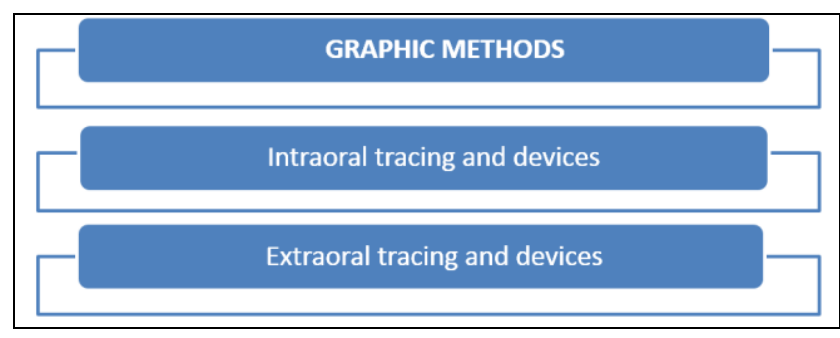

Intraoral Tracing Devices

- Cobletracer.

- Swiss dent ball bearing bitetracer'.

- Micro tracer.

Extraoral Tracing Devices

- Hight tracer.

- Searstracer.

- Phillips extraoral tracer.

\section{Description of the Arrow Point Tracing}

The device that provides a central point of bearing between the maxillary and mandibular arches. It consists of a contacting point attached to the maxillary arch and a plate attached to the opposing dental arch. The plate provides the even surface on which the bearing point slides as the jaw moves, though this tracing of the mandibular movement can be recorded. The advantage of using the plate and bearing point is to maintain and equalize the occlusal pressure.

\section{Central Bearing Device Consists of \\ - Central bearing point. \\ - Central bearing plate.}

\section{Types of Arrow Point Tracing}

Gerber stated that there are six different types of tracing obtained from Gothic arch tracers based on the direction of movement of condylar head and also on neuromuscular coordination of muscle of mastication and jaw movements. The various types of tracing obtained are as follows. Other than the below mentioned Gothic arch tracing forms, they can be further divided into Double Arrow Point, Interrupted Gothic Arch, and Atypical Form, based on the presence or absence of teeth. 

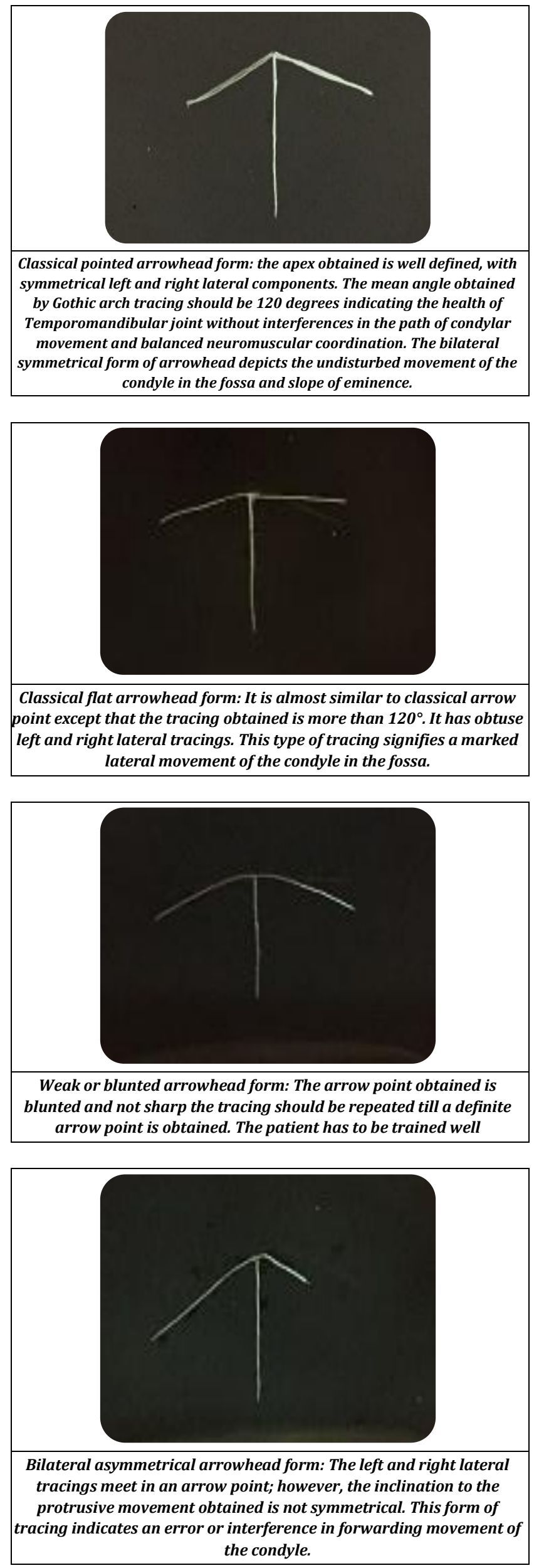

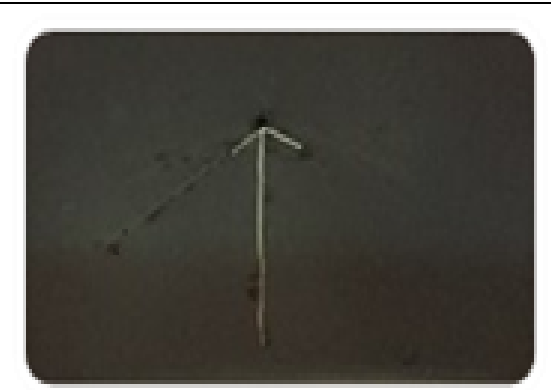

Small or miniature arrowhead point: Similar to the classical arrowhead point, however, the tracing obtained is small due to limited mandibular movement. This might be due to restricted neuromuscular coordination or mandibular movements or due to improper seating of record bases. It is also an indication of a being of edentulous for longer period of time with inhibition in mandibular movements

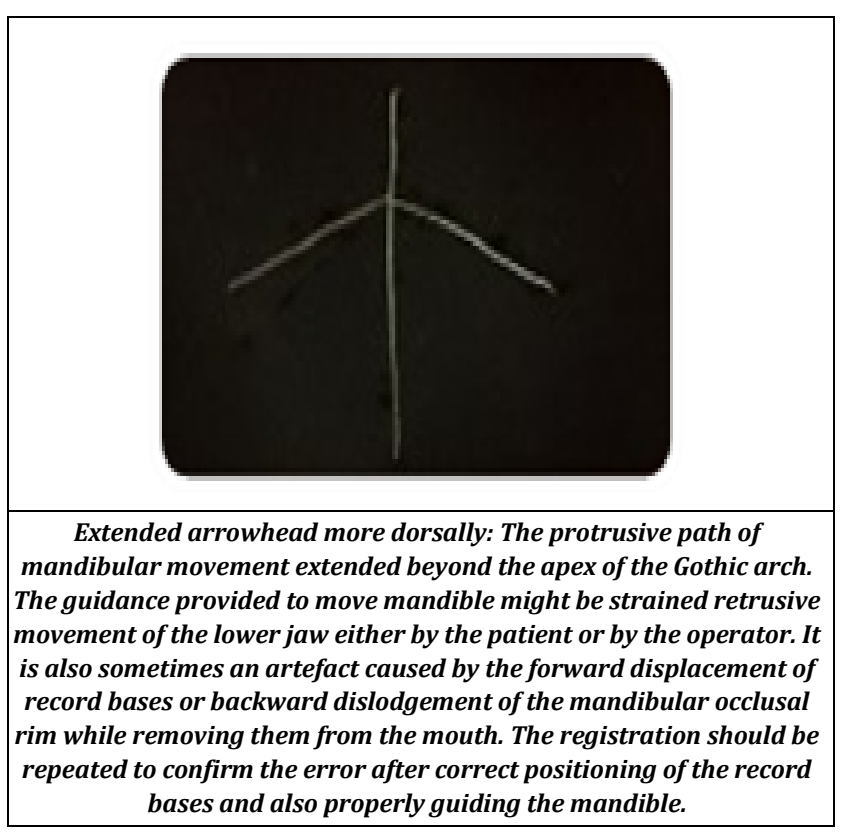

\section{Double Arrow Point}

It is a record with double arrowhead point obtained, caused due to habitual mandibular movement and retruded centric relation due to uncoordinated neuromuscular movement, patients should be trained and guided well.

\section{Interrupted Gothic Arch}

Break-in continuity of lateral path of a Gothic arch tracing obtained, due to interference or obstruction in movement caused due to touching of heels of occlusal rims during lateral movements.

\section{Atypical Form}

The component does not meet at the apex but on one of the lateral paths. This may happen in dentulous because of a faulty Muscular pattern due to par functional habits like bruxism. It is also seen in very old edentulous patients, who are using complete denture with incorrect centric relation. 


\section{Factors to be Considered While Making Records with Gothic Arch Tracers}

- The central bearing point has to be located in the centre to avoid pressure or displacement of the tissues or the records, due to unusual movement of the central bearing point when mandibular moves from centric to eccentric positions.

- The patient has to be guided to move the mandible in centric and all the eccentric positions to avoid the discrepancy or the minor errors.

- The central Bearing Point has to be located in the centre; utmost care has to be taken to place the device in the centre to equalize the pressure.

- Extraoral Graphic tracing methods, by visual means, supposed to be the most accurate method of making a centric and eccentric relation record; however, the graphic tracings method of recording centric and eccentric records are not necessarily precise. This record should be checked with an inter-oral record or with any means of computerized recording methods.

- The height of the alveolar ridges and the neuromuscular coordination of the jaws play an important role in recording various jaw movements.

- The denture base and the occlusal rims have to be well stabilized to accurately measure the jaw movements.

- Outmost care has to be taken while recording jaw movements in resorbed and flabby ridges to avoid errors caused due to a displacement of the tissues and the denture bases.

- It is difficult to stabilize a record base or central bearing device in patients with a large tongue as it might dislodge the tracers.

- $\quad$ Literature studies have confirmed that there will be $5 \%$ of error can be observed in the records whether made with intraoral graphic tracing or the extraoral graphic tracing records.

- The tracing is usually acceptable if the sharp apex is observed; if the tracing is not sharp then it is usually not acceptable. The blunt apex or the asymmetrical apex indicates that there is a discrepancy in the jaw movements following which it leads to error in recording centric relation.

- Graphic tracing is made with a predetermined vertical dimension, that harmonizes the Centric Relation at rest and centric relation at occlusion.

The major drawback of using graphic tracing records is The intra-oral and extra-oral tracing procedures were more complicated as compared to the other registration method. The tracers are contraindicated In patients with flabby ridges/highly resorbed ridges, or patients with loss of neuromuscular coordination[19],[20]

\section{Functional Recordings}

The functional method of recording centric relation was introduced in the year 1910, the techniques were introduced based on the principle of condylar movements in the centric and eccentric relationship (protrusive movement, retrusive movement, and right lateral and left lateral movement). Greene utilized a combination of pumice and plaster, amalgamated the materials placed it between the maxillary and mandibular occlusal rims, guided the mandible to do centric and the eccentric movements. These records were transferred to an articulator; the articulator was programmed based on the pattern obtained from a functional method of recording centric relation. Needle introduced 3 metallic studs, in 1923, these 3 studs were mounted onto maxillary occlusal rims made up of impression compound, patients were asked to do all the mandibular movements, based on the movements these studs grooved an arrow tracing into mandibular occlusal rims. These records were secured from the patient's mouth, following which the articulator was programmed with obtained functional grooves. House modified needles technique introduced 4 styli to make needlepoint tracings. Later on, Patterson, introduced modelling wax to make a record of centric relation, troughs were made in the maxillary and mandibular occlusal rims. Dental plaster was amalgamated with Carborundum, the mixture was then introduced into the trough[13],[17] The rims filled with troughs were placed in a patient's mouth, following which mandible was guided to move in centric and eccentric movements, until the desired curvature was been established. The major advantage of this technique would ensure uniform thickness and equalize the pressure in all the excursive movements. Modified functional technique, introduced by Meyer in 1934, in this technique soft wax was utilized to make occlusal rims, the occlusal surface of wax was coated with a thin tinfoil, following which it was lubricated, the rims were placed in patients mouth, following which mandible was guided to perform the functional movements to produce a path of movement as the jaws moved in the centric and all the eccentric movements. Later, plaster index was made on the wax rims. This plaster index was used as a guide to set the teeth. The instrument Gnathodynamo-meter by Boos was introduced in 1940, the instrument was used to determine the maximum occlusal force that could be recorded during the horizontal and vertical relationship of maxilla to mandibular arches. The biometer was mounted on the mandibular occlusal rim with a central bearing point against a plate that was mounted on the maxillary occlusal rim. The registrations with plaster were made with the biometer in the mouth and the readings were recorded while patients made exerted occlusal pressure. BOOS; advocated that the registration should be made under occlusal forces to determine the displacement of tissue under occlusal load while doing functional movements that would coincide with pressure made during occlusal registration. Shanahan. Introduced Physiologic technique in 1955, the cones made up of soft wax were mounted on the mandibular occlusal rim, following which patient was advised to make swallowing movements several times, while the tongue would force the mandible into its Centric Relation position. The cones of soft wax were moulded during these movements; hence the physiologic Centric Relation was recorded. Martin Henry Berman, in 1960 conducted a study "Accurate Inter Occlusal Records", he tested that the resistance of various inter-occlusal recording media, following which he concluded that: Accuracy of dental wax inter-occlusal records is questionable due to stress realising property and distortion. Zinc oxide eugenol impression paste has good flow property, that offers no resistance to closure and possesses many qualities favourable for obtaining the accurate interocclusal record. Kantor et al, in 1972 conducted a comparative investigation on Centric Relation recording 
techniques by considering the four different types of techniques to record centric relation i.e. swallowing method, chin point guidance technique, chin point guidance with anterior jig method and bilateral manipulation technique, later he summarised that: Bilateral manipulation produced the smallest area of displacement of the record when compared with the other recording techniques. The major disadvantage of using a functional technique of recording centric relation, the unstable bases can lead to dislodgement of the record bases in all the mandibular movement resulting in the inaccuracy of the centric relation records obtained. The tissue displacement will result due to the resistance caused due to recording medium, lack of neuromuscular control or equalized pressure in recording the eccentric relations contributes to inaccuracy in these methods. Patients need to have good neuromuscular coordination to participate in the functional methods of recording centric relations and also be capable of following instructions of the operator if accurate records are to be obtained. Bilateral manipulation showed the most consistent reproducibility and is recommended for recording Centric Relation. In 1974 Bilateral manipulation of the mandible to glide in centric relation was suggested by Peter Dawson.[12][13][18]. Lassila in 1986, conducted a study "Comparison of five inter-occlusal recording material" using silicone putty, polyether, zinc oxide eugenol impression paste, eugenol free zinc oxide, acrylic resin and baseplate wax, following which he concluded that: The initial resistance caused by various inter-occlusal recording material to closure changed from $0.5 \mathrm{~N}$ to $13.8 \mathrm{~N}$, a rapid rise in the working time was seen in all elastomers; Resistance caused by wax at $60^{\circ} \mathrm{C}$ was about $7 \mathrm{~N}$; Volumetric contraction of elastomers during polymerization was clinically minimal when compared with other materials. Dimensional stability of rigid material like acrylic resin and zinc oxide pastes were good. Elastomers maintained reliability for a relatively long period of time when stored in a tightly sealed plastic container. It is apparent from dental literature that with many opinions, thoughts and confusion concerning Centric Relation records, a certain technique might be required for an unusual situation. In the final analysis, it was concluded, that the skill of the operator and co-operation of the patient are probably the most important factors in securing an accurate Centric Relation record.

\section{Cephalometrics and Orthopantomography Techniques}

The use of radiographs in recording Centric Relation was described by Pyott and Schaeffer. Centric Relation and vertical dimension of occlusion can be determined by cephalometric radiographs. This method never gained widespread usage due to errors in recording centric relation. However, Orthopantomographs are the most commonly used radiographic technique to analyse and determine the condylar guidance angle.[15]The outline of the articular eminence and the glenoid fossa of the temporal bone can be traced and evaluated on the panoramic radiographs, to determine and measure the angle formed by condylar guidance. Two reference lines were drawn, the first reference line was Frankfort horizontal plane drawn on the right and left sides of the condyle. The second reference line was drawn by joining the most superior and most inferior point curvature of the glenoid fossa, this line was extended to intersect the Frankfort's horizontal plane to measure condylar guidance angle on the radiograph, the degree obtained by the angle should be proposed to be of valuable aid in setting the condylar guidance in semi-adjustable articulators. The use of the radiographic technique can overcome the shortcomings of the conventional clinical methods used to determine the condylar guidance. The limitations and drawbacks of using the radiographic technique should be precisely looked for errors and artefacts, the major disadvantage was the orientation of the reference plane, difficulty in distinguishing the articular eminence outline from the zygomatic arch. Further, the articular eminence inclination in the radiographic image was traced by a line joining the heights of curvature in the glenoid fossa and the articular eminence to represent the mean sagittal condylar path inclination.[15],[11] this may be different from the guiding inclination of protrusive movement of 4 to $6 \mathrm{~mm}$, which is the clinically significant range of protrusive movement of the mandible. Despite these drawbacks, the panoramic radiograph is useful for comparison of condylar guidance angles between right and left sides since it shows both the Temporomandibular joints with relatively same magnification errors. A radiographic error exists despite standardization. The clinical implication of these studies needs to be compared for reasonable accuracy by overcoming the errors caused due to superimposition of anatomical landmarks.

\section{Axiographic Technique}

The introduction of computerised means of obtaining the condylar guidance is gaining popularity in the field of dentistry. Recent advancements in computer technology have made it possible to analyse various mandibular movements with a high degree of resolution and precision. Beck and Morrison used an electromechanical device to reproduce mandibular motion in centric and eccentric movements, with the help of transducers, a recorder-controller, and a duplicator. Axiographic protrusive curves traced by hingeaxis reveal the movement of the condyle. The shape and range of movement of the axiographic tracings are useful. However, the axiographic tracings can be used to record centric and eccentric recordings that can be transferred to the fully adjustable articulator. [14][20] The major disadvantage of using the axiographic tracing method is such that the mandibular clutch is difficult to manipulate, it might lead to an error in recording, the limitation of utilizing axiograph is such that it cannot be used in patients with abnormal neuromuscular coordination and even in patients with severely resorbed ridges as it would lead to movement of record bases.

\section{CONCLUSIONS}

Recording of centric relation is the most important factor in dentistry. It is a very critical step. Therefore, it is recommended to discuss regarding various methods and material aspects used in recording centric relation more precisely. Skills of the operator and patient's good neuromuscular coordination are probably the most 
important factors in securing an accurate Centric Relation record.

\section{REFERENCES}

[1] Yeshwante B, Kale N, Baig N, et al. A path way to the centric - gothic arch. Int J of Allied Med Sci and Clin Res 2015;3(3):308-12.

[2] Bansal S, Palaskar J. Critical evaluation of methods to record centric jaw relation. J Indian Prosthodont Soc 2009;9(3):120-6.

[3] Singh S, Thombare R. Evaluating the validity of interocclusal plaster records made while recording protrusive jaw relation using extraoral tracing device. International Journal of Contemporary Dentistry 2011:7:2(3).

[4] Ferro KJ, Driscoll CF, Freilich MA, et al. Glossary of prosthodontic terms. The Journal of Prosthetic Dentistry 2017;117(5 Suppl):e1-105.

[5] Duggal N, Kadain P, Amardeep, et al. Meta-analysis of various methods of recording centric jaw relation - a literature review. Int J Health Sci Res 2017;7(3):341-50.

[6] Thakur M, Jain V, Parkash $\mathrm{H}$, et al. A comparative evaluation of static and functional methods for recording centric relation and condylar guidance: a clinical study. The Journal of Indian Prosthodontic Society 2012;12(3):175-81.

[7] Kumari VV, Anehosur GV, Meshramkar R, et al. An in vivo study to compare and correlate sagittal condylar guidance obtained by radiographic and extraoral gothic arch tracing method in edentulous patients. Eur J Prosthodont 2016;4:12-6.

[8] Patil R, Dubey S, Patil AK, et al. Correlation between sagittal condylar guidance obtained by gothic arch tracing an interocclusal record and by panoramic radiographic tracing in edentulous subjects: a clinicoradiographic analysis. IOSR Journal of Dental and Medical Sciences 2015;14(1):57-9.

[9] Sirana P, Kumar N, Malhotra A, et al. Comparative analysis of sagittal condylar guidance recorded by intraoral gothic arch tracing and panoramic radiograph in completely edentulous patients. The Journal of Contemporary Dental Practice 2018;19(11):1301-5.

[10] Maru K, Dwivedi A, Agarwal J, et al. Trends in selection, usage and techniques of interocclusal record materials among private dental practitioners: a survey.
Contemporary Clinical Dentistry 2018;9(Suppl 1):S127S32.

[11] Amin B, Kumar GPS, Raj B, et al. Assessment and comparison of the condylar guidance by protrusive interocclusal records and panoramic radiographic imaging in edentulous and dentulous individuals. Int J Prosthodont Restor Dent 2018;8(1):10-6.

[12] Gupta S, Tarannum F, Gupta NK, et al. Effect of head posture on tooth contacts in dentate and complete denture wearers using computerized occlusal analysis system. J Indian Prosthodont Soc 2017;17(3):250-4.

[13] Nandini VV, Nair KC, Sudhakar MC, et al. Comparative evaluation of hight tracer, chandra tracer, intraoral tracer, functiograph and checkbite: a clinical study. The Journal of Indian Prosthodontic Society 2005;5(1):26-32.

[14] Bansal S, Palaskar J. Critical evaluation of various methods of recording centric jaw relation. The Journal of Indian Prosthodontic Society 2008;8(4):185-91.

[15] Prasad KD, Prasad BR, Prasad AD, et al. Interocclusal records in prosthodontic rehabilitations-materials and techniques-a literature review. Nitte University Journal of Health Science 2012;2(3):54-60.

[16] Gupta M, Barabde A, Thombare R, et al. Clinicoradiographic analysis of horizontal condylar guidance determined by hight tracer, novel indigenous intraoral digitracer and checkbite in complete denture prosthesis. International Journal of Current Research 2017;9(5):49940-6.

[17] Shetty S, Kunta M, Shenoy K. A clinico-radiographic study to compare and co-relate sagittal condylar guidance determined by intraoral gothic arch tracing method and panoramic radiograph in completely edentulous patients. J Indian Prosthodont Soc 2018;18(1):19-23.

[18] Watanabe Y. Use of personal computers for gothic arch tracing: analysis and evaluation of horizontal mandibular positions with edentulous prosthesis. The Journal of Prosthetic Dentistry 1999;82(5):562-72.

[19] Podesta RE, Radke RA, Fullmer FD, et al. Comparative study and analysis of mandibular movements utilizing dynamic recording systems. Journal of Gnathology 1997;16(1):61-9.

[20] Prasad KD, Shetty M, Chandy BK. Evaluation of condylar inclination of dentulous subjects determined by axiograph and to compare with manual programming of articulators using protrusive interocclusal record. Contemporary Clinical Dentistry 2015;6(3):371-4. 